\title{
Gamma/Hadron Separation for a Ground Based IACT in Experiment TAIGA Using Random Forest Machine
}

\section{Learning Methods}

Maria Vasyutina, ${ }^{a}, *$ Lyubov Sveshnikova, ${ }^{a}$ I. I. Astapov, ${ }^{b}$ P. A. Bezyazeekov, ${ }^{c}$ M. Blank, ${ }^{d}$ E. A. Bonvech, ${ }^{a}$ A. N. Borodin, ${ }^{e}$ M. Brueckner, ${ }^{f}$ N. M. Budnev, ${ }^{c}$ A. V. Bulan, ${ }^{a}$ D. V. Chernov, ${ }^{a}$ A. Chiavassa, ${ }^{g}$ A. N. Dyachok, ${ }^{c}$ A. R. Gafarov, ${ }^{c}$ A. Yu. Garmash, ${ }^{h, i}$ V. M. Grebenyuk, ${ }^{e}$ O. A. Gress, ${ }^{c}$ T. I. Gress, ${ }^{c}$ A. A. Grinyuk, ${ }^{e}$ O. G. Grishin, ${ }^{c}$ D. Horns, ${ }^{d}$ A. L. Ivanova, ${ }^{c, i}$ N. N. Kalmykov, ${ }^{a}$ V. V. Kindin, ${ }^{b}$ S. N. Kiryuhin, ${ }^{c}$ R. P. Kokoulin, ${ }^{b}$ K. G. Kompaniets, ${ }^{b}$ E. E. Korosteleva, ${ }^{a}$ V. A. Kozhin, ${ }^{a}$ E. A. Kravchenko, ${ }^{h, i}$ A. P. Kryukov, ${ }^{a}$ L. A. Kuzmichev, ${ }^{a}$ A. A. Lagutin, ${ }^{j}$ M. V. Lavrova, ${ }^{e}$ Yu. Lemeshev, ${ }^{c}$ B. K. Lubsandorzhiev, ${ }^{k}$ N. B. Lubsandorzhiev, ${ }^{a}$ A. D. Lukanov, ${ }^{k}$ D. Lukyantsev, ${ }^{c}$ R. R. Mirgazov, ${ }^{c}$ R. Mirzoyan, ${ }^{a, l}$ R. D. Monkhoev, ${ }^{c}$ E. A. Osipova, ${ }^{a}$ A. L. Pakhorukov, ${ }^{c}$ L. A. Panasenko, ${ }^{h}$ A. Pan, ${ }^{e}$ L. V. Pankov, ${ }^{c}$ A. D. Panov, ${ }^{a}$ A. A. Petrukhin, ${ }^{b}$ D. A.

Podgrudkov, ${ }^{a}$ V. A. Poleschuk, ${ }^{c}$ M. Popesku, ${ }^{m}$ E. G. Popova, ${ }^{a}$ A. Porelli, ${ }^{f}$ E. B. Postnikov, ${ }^{a}$ V. V. Prosin, ${ }^{a}$ V. S. Ptuskin, ${ }^{n}$ A. A. Pushnin, ${ }^{c}$ R. I. Raikin, ${ }^{j}$. Razumov, ${ }^{a}$ E. Rjabov, ${ }^{c}$ G. I. Rubtsov, ${ }^{k}$ Y. I. Sagan, ${ }^{e, o}$ V. S. Samoliga, ${ }^{c}$ A. Yu. Sidorenkov, ${ }^{k}$ A. A. Silaev, ${ }^{a}$ A. A. Silaev (junior), ${ }^{a}$ A. V. Skurikhin, ${ }^{a}$ M. Slunecka, ${ }^{e}$ A. V. Sokolov, ${ }^{h, i}$ Y. Suvorkin, ${ }^{c}$ V. A. Tabolenko, ${ }^{c}$ A. B. Tanaev, ${ }^{c}$ B. A. Tarashansky, ${ }^{c}$ M. Ternovoy, ${ }^{c}$ L. G. Tkachev, ${ }^{e, o}$ M. Tluczykont, ${ }^{d}$ N. Ushakov, ${ }^{k}$ A. Vaidyanathan, ${ }^{h}$ P. A. Volchugov, ${ }^{a}$ N. V. Volkov, ${ }^{j}$ D. Voronin, ${ }^{k}$ R. Wischnewski, ${ }^{f}$ I. I. Yashin, ${ }^{b}$ A. V. Zagorodnikov ${ }^{c}$ and D. P. Zhurov $^{c}$

\footnotetext{
${ }^{a}$ Lomonosov Moscow State University Skobeltsyn Institute of Nuclear Physics (MSU SINP), Leninskie gory 1(2), GSP-1, Moscow, 119991, Russia.

${ }^{b}$ National Research Nuclear University MEPhI (Moscow Engineering Physics Institute), Kashirskoe highway 31, Moscow, 115409, Russia

${ }^{c}$ Institute of Applied Physics, Irkutsk State University (API ISU), Gagarin Blvd. 20, Irkutsk,664003, Russia

${ }^{d}$ Institute of experimental physics of Hamburg University, Luruper Chaussee 149, 22761, Hamburg, Germany

e Joint Institute for Nuclear Research, Joliot-Curie 6, Dubna, Moscow Region, 141980, Russia

${ }^{f}$ DeutschesElektronen-Synchrotron DESY, 15738, Zeuthen, Germany
}

*Speaker 
${ }^{g}$ Physics Department of the University of Torino and the National Institute of Nuclear Physics INFN, 10125, Torino, Italy

${ }^{h}$ Novosibirsk State University, Pirogova 1, Novosibirsk, 630090, Russia

${ }^{i}$ Budker Institute of Nuclear Physics of the Siberian Branch of the Russian Academy of Sciences, Lavrentyev Prosp. 11, Novosibirsk, 630090, Russia

${ }^{j}$ Altai State University,

Lenina 61, Barnaul, 656049, Russia

${ }^{k}$ Institute for Nuclear Research of the Russian Academy of Sciences, 60th October Anniversary 7a, 117312, Moscow, Russia

${ }^{l}$ Max Planck Institute for Physics,

Fohringer Ring 6, D-80805 Munich, Germany

${ }^{m}$ Institute of Space Science,

P.O.Box MG-23, Ro 077125, Bucharest-Magurele, Romania

${ }^{n}$ Pushkov Institute of Terrestrial Magnetism, Ionosphere and Radio Wave Propagation of the Siberian Branch of the Russian Academy of Sciences (IZMIRAN),

Kaluzhskoe highway 4, Moscow, Troitsk, 108840, Russia

${ }^{o}$ Dubna State University,

Universitetskaya 19, Dubna, Moscow region, 141982, Russia

E-mail: masha.vasutina.1998@gmail.com, tfl10@mail.ru

In this paper we present the first attempt of adaptation the Random Forest (RF) machine learning algorithm to gamma/hadron separation in the TAIGA experiment (Tunka Advanced Instrument for cosmic ray physics and Gamma-ray Astronomy). The TAIGA experiment will include HiSCORE array with 120 wide-angle Cherenkov detectors on the area of $1 \mathrm{~km}^{2}$ and 5 Imaging Atmospheric Cherenkov Telescopes (IACT) on the same area. At the first stage of the analysis, only images obtained by one IACT were included in consideration. The training process occurs on samples of parameterized images obtained from Monte Carlo (MC) data for gammas and hadrons with a 'Scaled Hillas Parameters' standard technique. It was shown that the program effectively separates gamma-like showers, RF method does produce stable results and is robust with respect to input parameters and provides a simple control and setup of the procedure for extracting showers from gamma rays.

The 5th International Workshop on Deep Learning in Computational Physics 28-29 June, 2021

Moscow, Russia 


\section{Introduction}

The important goal of Very High Energy (VHE) gamma ray astronomy is to detect gamma rays with energy around and more than $100 \mathrm{TeV}$, that requires a development of new arrays with very large observation area (by the order of $1 \mathrm{~km}^{2}$ and more) [1-3] because of tiny flux of high energy (HE) gamma rays. In addition, it is necessary to distinguish these gamma quanta against the background of cosmic rays, the flux of which is $10^{4}$ times higher. One of the possible approaches (hybrid method) was proposed and realized several years ago [4-6] in the experiment TAIGA (Tunka Advanced Instrument for cosmic ray physics and Gamma Astronomy). This array is located in Tunka Valley, $50 \mathrm{~km}$ off the Lake Baikal. A first part of array TAIGA has been deployed and at present consists of 110 wide -angle stations of timing array TAIGA-HiSCORE and 3 IACTs (Imaging Atmospheric Cherenkov Telescopes). The timing electronic allows one to measure a time of arriving Cherenkov photons from atmospheric shower with nanosecond accuracy, that in turn allows one to reconstruct the arriving direction of showers with accuracy 0.1-0.4 degrees [7], and also to measure an energy and core positions of showers. Exploitation of HiSCORE array reveals a fairly high enough threshold of gamma ray registration, $40 \mathrm{TeV}$, that practically does not allow to carry out calibrating measurement using the Crab 'standard candle'. The 'imaging' part of the installation, 3 IACTs, were included in the array in order to improve radically the background suppression efficiency because the images of atmospheric Cherenkov showers detected by IACT are sensitive to the type of primary particles. As a result, every detected event is characterized by a huge number of parameters, obtained from all detectors. And the need to adapt, develop and to apply machine learning and classification methods for data analysis became clear. At this first stage of the analysis, only images obtained by one IACT were included in consideration to check the possibility of implementation, usage, and functionality of the classification Random Forest algorithm for the given experimental data (in this work, we used the Random Forest Classifier function from the library Scikit Learn).

On the Figure1 the telescope IACT01 and the example of an image, detected by the telescope are presented. Details of construction can be found in [7].
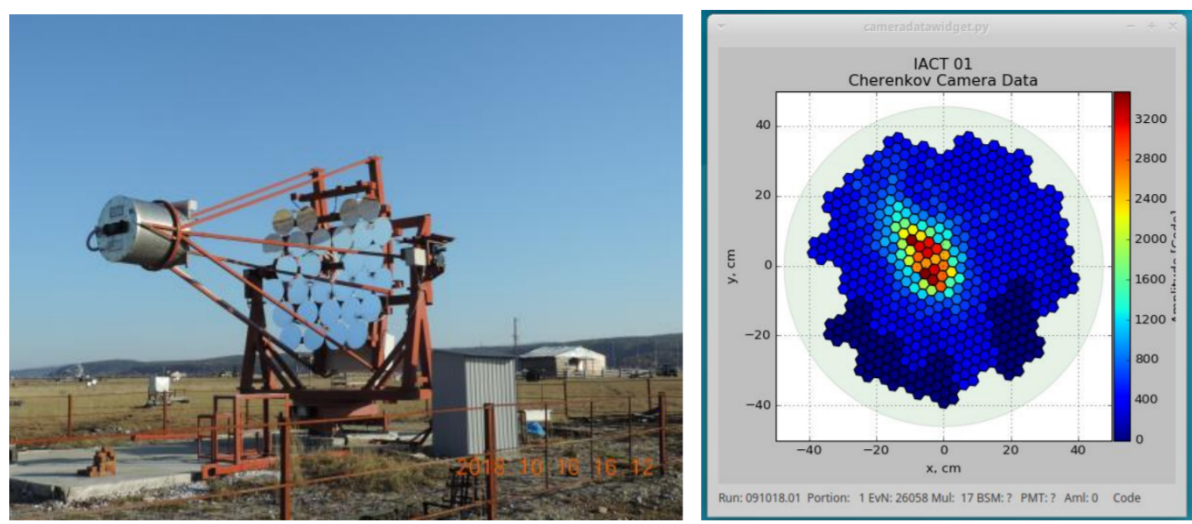

Figure 1: The first telescope and the example of detected image in the TAIGA observatory.[7] 


\section{Experimental samples and Monte-Carlo training samples}

As an experimental sample for the analysis, we have chosen the data detected with the IACT01 in TAIGA experiment during the October, November and the first part of December 2019 year at pointing to the source Crab Nebula (Crab - 'standard candle source' in TeV gamma astronomy), published in [8]. In the TAIGA experiment a 'wobbling' tracking system of the telescope was realized (see details in [9]): Experimental sample consists of 'On' and 'Off' samples. The first one corresponds to the case when telescope follows the source in the sky, the second one, when telescope follows a background point in the sky. Every event is characterized by $\sim 15$ parameters. Some of them represents the overall characteristics of image: Size - full brightness (full number of photoelectrons), $N \_p x$ - number of hit pixels, dens - mean photon density, Con 2 - photon concentration in image. Other parameters (so called Hillas parameters [10] characterize a position, a direction, a width and a length of the image detected in the chamber (see Figure 1). In our method some parameters differ for 'On' and 'Off' modes: dist (distance between the position of weighted center of image (WC) and position of source pixel) and alpha (the angle between the line directed to the source pixel and major axis of Hillas's ellipse).

In this work we use additionally so-called scaled parameters [11], which allow to exclude the size dependence of the width and length parameters. The size range of MC data is divided into bins, and for each bin 'i' we calculate a mean value of $<w_{i d t} h_{g}^{i}>$ and variance $S_{i g} g_{g}^{i}$ for gamma rays. For the every event width $h^{i}$ is transformed to scaled value $w i d t h_{s c}^{i}$ by the following formulae:

$$
w_{i d t h}^{i}=\frac{w_{s c} d t h^{i}-<w i d t h_{g}^{i}>}{\operatorname{Sig}_{g}^{i}}
$$

As a result, we have a normalized width and length distribution for gammas: they follow with mean value 0 and variance 1 for gamma quanta and two-three times larger for hadrons.

The full MC simulation has been done (see details in $[12,13]$ ), taking into account the design of the installation, the optical system of the telescope, the trigger system for collecting data for the background showers (originated from protons, helium nuclei) and showers from gamma quanta. Basing on background 'Off' events a complete coordination of the different experimental and MC image parameters has been carried out.

Our experimental final sample, selected for the analysis is 650000 events. Basing on these data, an excess of gamma quanta from the Crab was observed in the TAIGA experiment in the energy range of 3-30 TeV with a significance of about 5 sigma [8]. The effective cuts for gamma/background separating were selected manually, based on the distributions of various parameters obtained in MC simulations for gamma quanta and hadrons. Parameter alpha was used only on the latest stage, as the most powerful and visual parameter for selecting gamma quanta (see Figure 2), because of background events should have more or less uniform distribution by alpha in contrast to gamma induced image concentrated up to alpha $<10$ degrees.

Further we also will exclude the alpha from criteria to check the new approach.

The Random Forest method is based on a collection of decision trees, and, like many other classification and regression methods, a Random Forest is constructed on the basis of training 


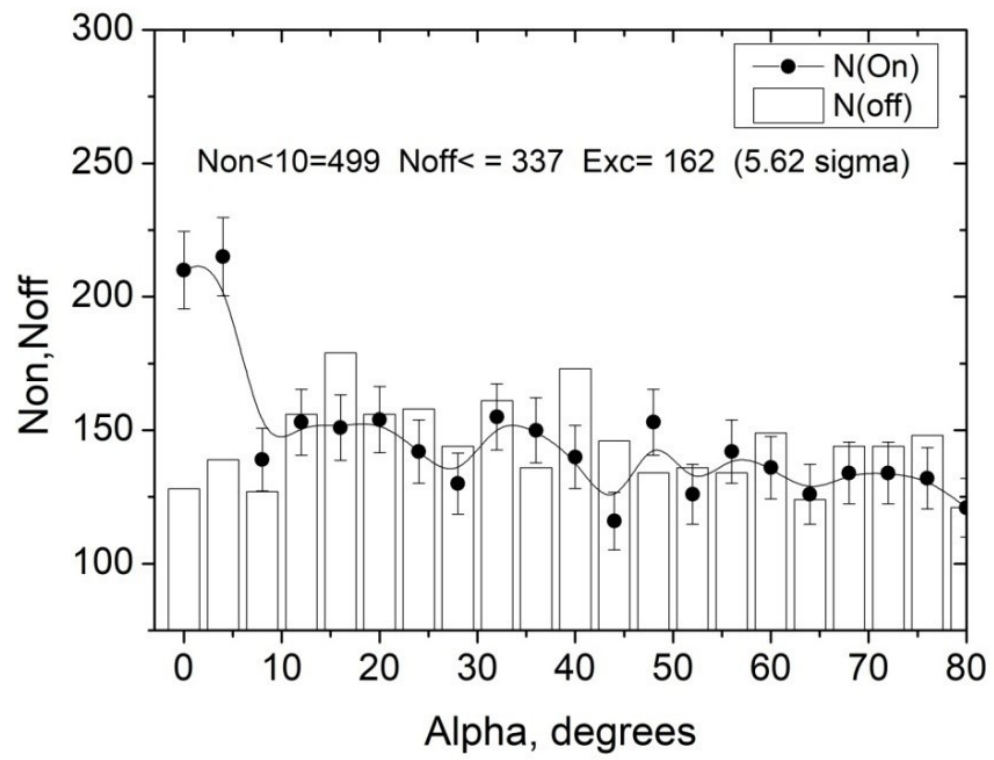

Figure 2: Distribution by parameter alpha for source ('On') events and background ('Off') events detected from Crab with following cuts: Size $>125$ p.e.; dist= 0.36 o $-1.44 \mathrm{o}, 0.024 \mathrm{o}<$ Width $<0.068 \mathrm{o} \times(\operatorname{lgSize}-0.047)$, Length $<0.31$ o, Con $>0.54$ [8]

samples obtained usually by Monte-Carlo method (MC). MC samples should be calculated as similar as possible to experimental samples. It is not a simple task due to a complexity of experimental data, as usually included many methodical details. So sometimes for the purpose of $\mathrm{g} / \mathrm{h}$ separation, the training samples for hadrons is selected from experimental 'Off' data. These two these cases will be applied in this paper. Difference in gamma-like events obtained as a result of classification for 'On' and 'Off' experimental samples is considered as an excess of gamma quanta.

\section{Random Forest method}

We use the Random Forest method and closely follow the method described by L. Breiman [14], and at the first stage we repeat the approach realized in the MAGIC experiment for gamma/hadron separation [11].

The Random Forest method is based on an ensemble of decision trees. The main step of the method is a construction of the training and tested samples. In our case the training MC sample includes two classes (gamma and hadrons) of events (vectors of image parameters described in previous chapter). One binary decision tree can be constructed from this sample (root node), than it is subdivided into two classes by one randomly chosen parameter (in our case we use more complicated choice, see below). To measure the separation ability of an image parameter and to optimize the cut value in the given node the Gini index is used in our program [11]. Choosing the smallest $Q_{\text {Gini }}$ corresponds to minimizing the variance of the population of gammas and hadrons in the subsequent nodes. Minimization of the Gini index provides both the choice of the image 
parameter and the split value to be used. In our case for two classes the Gini index is calculated based on $N_{g}, N_{h}$ in the given node and the total number events in the node $\mathrm{N}$ [11]:

$$
Q_{G i n i}=1-\left(\frac{N_{g}}{N}\right)^{2}-\left(\frac{N_{h}}{N}\right)^{2}
$$

This procedure subsequently is repeated, the number of nodes doubles as the number of layers increases. The procedure stops, if there are approximately only one class of events left in the node. In our application we used modification of the program with calculation of the mean hadronness instead of a 0 or 1 majority vote for a class, as in [11]. For every terminal node with $N_{g}$ and $N_{h}$ in this node the parameter $h$ (hadroness) is calculated, which is assigned to all events in this terminal node:

$$
h=\frac{N_{h}}{N_{h}+N_{g}}
$$

It is possible also to limit the full depth of the tree. The example of the decision tree with maximal depth 3 is presented in Figure 3.

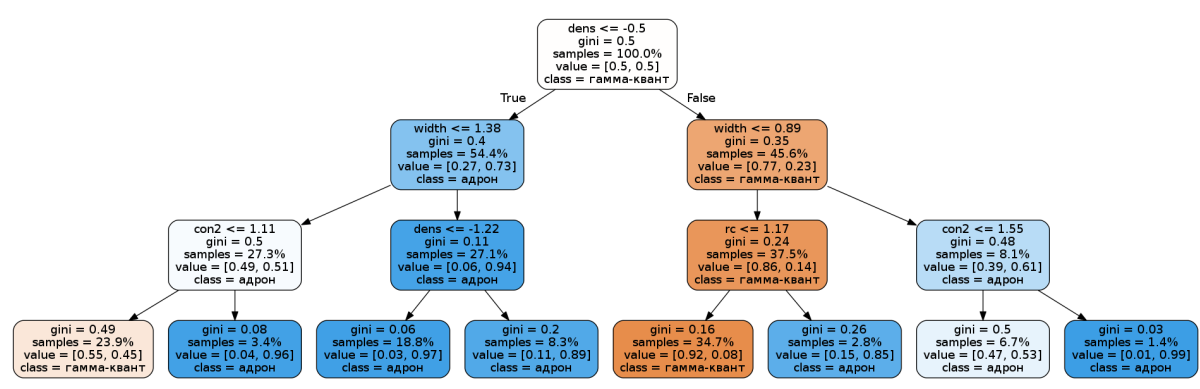

Figure 3: Example of one decision tree with 3 layers. Blue nodes have mostly hadrons, the red ones have most gamma quanta, other nodes have a mixed composition.

The accuracy of classification with one tree is not good enough, so the procedure of classification is carried out usually with many trees (in our program the optimal value occurred to be around 100 trees), that dramatically improves the separation ability of the method. To obtain independent trees with the same training sample a 'bootstrap sample' [11] is used for each tree by sampling $\mathrm{n}$ times with return from the original training sample, which contains $\mathrm{n}$ events. This procedure guarantees that the event image parameter distributions are statistically identical, but in each bootstrap sample there will be on average $(1-1 / \mathrm{e})$ original training events, the rest events are copies [11]. If we carry out $N_{\text {trees }}$ the final hadronness for the every 'i' event can be recalculated as mean value averaged by all trees.

$$
<h_{i}>=\sum \frac{h_{i}}{N_{\text {trees }}}
$$


The example of distribution of all events by this parameter is presented in Figure 4 from [11] for the case of equal number of primary gamma-quanta and hadrons in the training sample.

The distribution by hadronness depends on many factors, the better algorithm, the narrower peaks for gamma quanta (left) and for hadrons (right) should appear. This is a very important parameter, because it indicates if the event belongs to the class of gammas or hadrons. It seems natural to introduce one boundary, $H_{\text {cut }}$, dividing the selected events into two classes: gamma and hadrons at $H_{\text {cut }}=0.5$. But in our case the classes are not equivalent because of the tiny flow of gamma quanta in the cosmic ray stream, and it is necessary to distinguish gamma quanta well, so this value requires optimization.

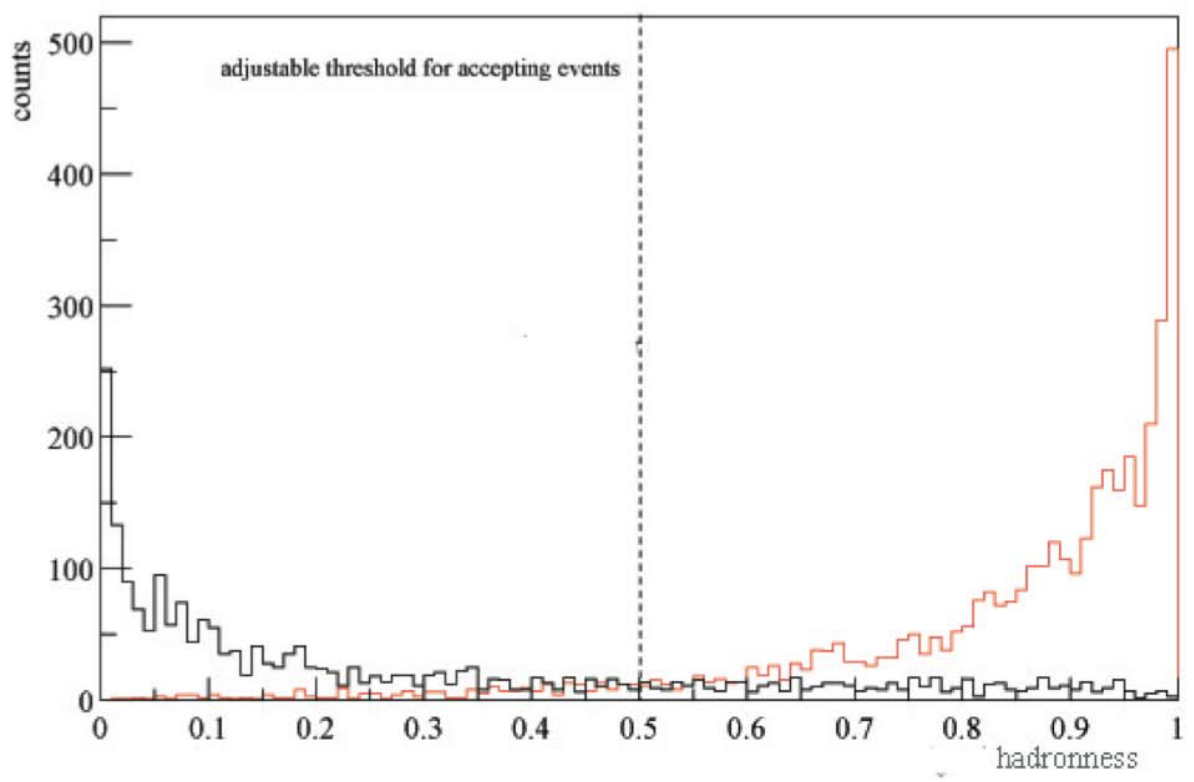

Figure 4: Example of hadronness distribution for the gamma sample (black lines) and for the hadron sample (red lines) after RF classification [11]

\section{Results of classification obtained with the training and test samples}

Here we tune the RF program parameters based on the training MC samples. Main of these parameters directly determine the final results and accuracy. We have found optimal values of the number of trees and depth of trees, number of trials in random split selection, number of events in terminal nodes, types of parameters which effectively take part in RF classification, the effective criteria for successfulness of classification necessary for our scientific tasks.

As the main criteria of successfulness of classification we have chosen 3 main parameters: $Q_{\text {factor }}$ is widely used in gamma astronomy for estimation of gamma quanta extracting efficiency from hadron background; $\varepsilon_{g}$ (a part of primary gamma-quanta classified as gamma), $\varepsilon_{h}$ (a part of primary hadron events classified as gamma, sometimes it is called 'background suppression efficiency') Below we present the way of calculation of these three values, where $\mathrm{N}(1 \rightarrow 2)$ means the number of primary events of class 1 which were classified during the RF procedure as events of class 2: 


$$
\begin{gathered}
\varepsilon_{g}=\frac{N(g->g)}{N(g->g)+N(g->h)} \\
\varepsilon_{h}=\frac{N(h->g)}{N(h->h)+N(h->g)} \\
Q_{f a c}=\frac{\varepsilon_{g}}{\sqrt{\varepsilon_{h}}}
\end{gathered}
$$

Primary gamma training sample contains $67767 \mathrm{MC}$ simulated showers in the interval 1-50 TeV distributed in accordance with a power-law spectrum $\sim E^{-2.6}$. This sample was was reduced by criteria Size $>60$ ph.e. (photoelectrons) and dist <23 (see chapter 2). The final sample of gamma quanta contains 440000 events. Primary hadron training file contains 484000 simulated showers [12] produced by primary protons and helium in the interval 2-100 TeV with the same slope. The final hadron sample contains 140000 events with Size> 60 ph.e. and dist<23 (see chapter 2). For every tree we have generated bootstrap sample containing the equal number of gammas and hadrons 18438. The test sample generated by the same way, consists of 4609 hadrons and 4609 gamma events. At the first step of analysis two very important program parameters - the optimal number of trees and parameter $H_{c u t}$ (a main classificator in our approach) were considered. In the Table 1 the dependence of $\varepsilon_{g}, \varepsilon_{h}, Q_{f a c}$ on the number of trees is presented for two values of limit $H_{c u t}$.

\begin{tabular}{|c|c|c|c|c|c|c|}
\hline & \multicolumn{3}{|c|}{$H_{\text {cut }}<0.5$} & \multicolumn{3}{c|}{$H_{\text {cut }}<0.05$} \\
\hline N trees & $\varepsilon_{g}$ & $\varepsilon_{h}$ & $Q_{\text {fac }}$ & $\varepsilon_{g}$ & $\varepsilon_{h}$ & $Q_{\text {fac }}$ \\
\hline 1 & 0.79 & 0.137 & 2.13 & 0.79 & 0.137 & 2.13 \\
\hline 10 & 0.86 & 0.123 & 2.45 & 0.44 & 0.017 & 3.32 \\
\hline 100 & 0.85 & 0.109 & 2.57 & 0.38 & 0.096 & 3.88 \\
\hline 1000 & 0.85 & 0.110 & 2.57 & 0.39 & 0.011 & 3.75 \\
\hline 10000 & 0.85 & 0.111 & 2.57 & 0.39 & 0.011 & 3.75 \\
\hline
\end{tabular}

Table 1: Dependence of $Q_{f a c}$ on the number of trees and on limit of parameter $H_{c u t}$

$Q_{f a c}$ value is very sensitive to the number of trees only at the small number of trees, and the difference in $Q_{\text {fac }}$ at $N_{\text {trees }}>100$ is practically absent. $N_{\text {trees }}=100$ will be used in further analysis.

The absolute value of $Q_{f a c}$ for the $H_{c u t}=0.05$ is obviously larger than for the standard value $H_{c u t}=0.5$. To search for the optimal $H_{c u t}$ parameter in the Table 2 the more detailed dependence $Q_{\text {fac }}\left(H_{c u t}\right)$ is presented. $H_{c u t}=0.05$ will be used in further analysis.

Every event is characterized by a vector of image parameters, but not all of them are identical for classification. Some of them are very sensitive to the type of primary particles: width, length, con2, dens. They must be included in the procedure of RF classification (we call them RF parameters). But Size determines the energy threshold, and dist parameter determines the effective radius of shower collection around the telescope. This is a reason why sometimes we introduce the cuts by 


\begin{tabular}{|l|c|l|c|}
\hline$H_{\text {cut }}$ & $\varepsilon_{g}$ & \multicolumn{1}{|c|}{$\varepsilon_{h}$} & $Q_{\text {fac }}$ \\
\hline 0.2 & 0.652 & 0.042 & 3.182 \\
\hline 0.15 & 0.595 & 0.030 & 3.385 \\
\hline 0.10 & 0.512 & 0.019 & 3.658 \\
\hline 0.05 & 0.382 & 0.0096 & 3.889 \\
\hline 0.02 & 0.244 & 0.0 .005 & 3.430 \\
\hline
\end{tabular}

Table 2: Dependence of $Q_{f a c}$ on the limit of parameter 'hadronness', $H_{c u t}$

these parameters before RF classification. In the Table 3 and Table 4 the dependence of $Q_{f a c}$ on the threshold value of image size and dist is presented.

\begin{tabular}{|l|c|c|l|}
\hline Size & $\varepsilon_{g}$ & $\varepsilon_{h}$ & $Q_{\text {fac }}$ \\
\hline 60 & 0.280 & 0.013 & 2.45 \\
\hline 125 & 0.382 & 0.0096 & 3.89 \\
\hline 140 & 0.394 & 0.0091 & 4.12 \\
\hline 150 & 0.406 & 0.0079 & 4.57 \\
\hline 160 & 0.412 & 0.0099 & 4.14 \\
\hline 180 & 0.432 & 0.0085 & 4.67 \\
\hline
\end{tabular}

Table 3: Dependence of $Q_{f a c}$ on the threshold value of image size

\begin{tabular}{|l|c|c|l|}
\hline dist, $\mathrm{cm}$ & $\varepsilon_{g}$ & $\varepsilon_{h}$ & $Q_{\text {fac }}$ \\
\hline 23 & 0.38 & 0.0096 & 3.89 \\
\hline 18 & 0.34 & 0.0089 & 3.62 \\
\hline 13 & 0.33 & 0.0063 & 4.16 \\
\hline 8 & 0.46 & 0.0097 & 4.67 \\
\hline 5 & 0.39 & 0.0023 & 8.09 \\
\hline
\end{tabular}

Table 4: Dependence of $Q_{f a c}$ on the threshold value of of parameter dist for events with size $>125$ ph.e.

Based on these results we have chosen optimal values of threshold size in the interval 125-150 ph.e. and dist in the interval dist $<13 \mathrm{~cm}$ for further analysis. The final way of random split selection in every node was as follows. All parameters width, length, dens, con2, dist, size were used in optimization at the given nod. From them, only one parameter with minimal $Q_{\text {Gini }}$ index is used for splitting events onto two classes. It is necessary to emphasize that up to now we did not include in the vector of parameters the parameter alpha.

\section{Results of classification obtained in experiment}

A full primary experimental sample being analyzed in this work consists of 1500000 events. As it is described in the chapter 2, these events were detected with the IACT01 in experiment 
TAIGA during the October, November and the first part of December 2019 year at pointing the source Crab Nebula (Crab - 'standard candle source' in TeV gamma astronomy). The first result of observation of gamma quantum from Crab in experiment TAIGAs was published in [8]. This result was shown in Figure 2. As it was mentioned in chapter 2 experimental sample is divided into 'On' and 'Off' samples. The 'On' sample corresponds to the case when telescope follows the source in the sky, and we should observe some excess of gamma events from Crab in this sample. In the 'Off' sample one can expect only fake gamma quanta. Since background events have more or less uniform distribution by alpha in contrast to gamma induced image, concentrated up to alpha $<10$ degrees, the excess of gamma quanta is calculated as

$$
\text { Excess }=N_{O N}\left(\text { alpha }<10^{\circ}\right)-N_{O F F}\left(\text { alpha }<10^{\circ}\right)
$$
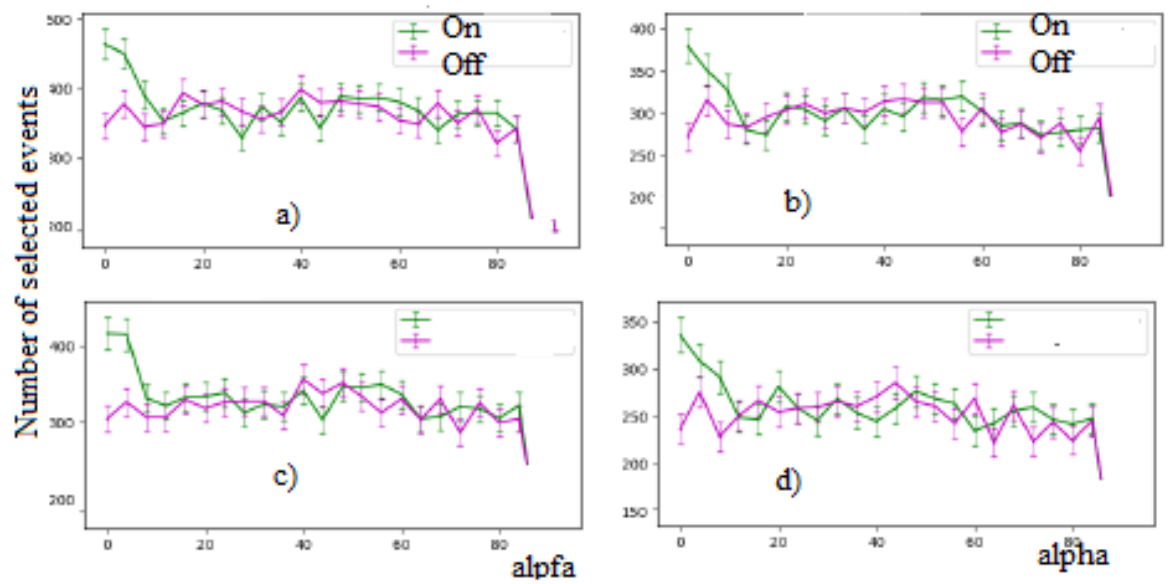

Figure 5: : Distribution of parameter alpha, obtained after RF classification of the experimental sample 'On' and experimental sample 'Off' for different Size threshold: (a) Size >125 ph.e., (b) Size >150 ph.e. with fixing these parameters as input threshold for RF classification; c) Size $>125$ p.e. + Size as parameter included in classification; d) Size $>150$ p.e. + Size as parameter included in classification.

After excluding the events with Size $<60$ ph.e. and dist $>23 \mathrm{~cm}$, the final sample contains 666462 events. After introduction of a cut on Size $>125$ ph.e., only 378515 are left, and at cut Size $>150$ ph.e. statistics decrease to 315220 events. Both these samples were used as test samples for procedure of Random Forest classification. In the next Figure 5 we present the variants of calculations, when RF classification was carried out without parameter alpha. This case is most close to the Figure 2, obtained in experiment TAIGA by manual way. Numerical values of Excess are shown in the Table 5.

We can compare the best results in Table 5 and Figure 5 (case a), obtained by Random Forest method, with Figure 2, where it was obtained manually. It is seen from the Figure 5 that Random Forest method gives the results very similar to the results, obtained in the experiment TAIGA on the same experimental data sample. Larger value of excess (237 against 162) and slightly less 


\begin{tabular}{|c|c|c|c|l|l|}
\hline & Size & $\begin{array}{c}N_{O n} \\
(\mathrm{alpha<10)}\end{array}$ & $\begin{array}{c}N_{\text {Off }} \\
(\text { alpha< } 10)\end{array}$ & Excess (alpha<10) & Significance \\
\hline b) Size as threshold & $>150$ p.e. & 1059 & 875 & 184 & 4.18 \\
\hline a) Size as threshold & $>\mathbf{1 2 5}$ p.e. & $\mathbf{1 3 1 6}$ & $\mathbf{1 0 6 9}$ & $\mathbf{2 3 7}$ & $\mathbf{4 . 8}$ \\
\hline $\begin{array}{c}\text { d) Size as parameter } \\
\text { and as threshold }\end{array}$ & $>150$ p.e. & 937 & 742 & 195 & 4.75 \\
\hline $\begin{array}{c}\text { c) Size as parameter } \\
\text { and as threshold }\end{array}$ & $>125$ p.e. & 1163 & 939 & 224 & 4.8 \\
\hline
\end{tabular}

Table 5: Results of application of RF method for classification of experimental events.

significance (4.8 sigma against 5.6 sigma in Figure 2, is connected with the fact that parameter dist was not limited as in [8]. It resulted to the larger radius of shower collection.

\section{Conclusions}

In TAIGA experiment the first attempt of application of Random Forest algorithm for the selection of tiny flow of high energy gamma rays above a very large flow of cosmic ray's background has shown the efficiency of RF approach.

1. Based on the training samples, obtained from the Monte Carlo data for primary gamma quanta and hadrons, the optimal settings of the program parameters, such as the number of trees, the depth of trees, an optimization of event parameters, criteria of efficiency of classification and other technical details, were searched for and established. It is shown that the method produces stable results and is robust to input parameters.

2. A similar optimization was carried out on the experimental samples ' $\mathrm{ON}$ ' (when the telescope is directed to the source) and 'OFF' (when the telescope is directed to the background of the sky). The dependence on the alpha parameter showed the possibility of separating events initiated by gamma quanta from the background of hadrons.

3. The obtained result is compared to the method of background suppression by semi-empirical selection of parameters used in the TAIGA experiment. It is shown that RF method gives the similar number of selected gamma quanta, but RF method requires much less efforts for the analysis. The much simpler control and tuning of the gamma-ray shower extraction procedure allows to hope to use this method for multidimensional analysis of events, obtained by hybrid method in TAIGA experiment.

\section{Acknowledgment}

The work was performed at the unique scientific installation "Astrophysical Complex of MSUISU"(agreement 13.UNU.21.0007). This work also is supported by the Russian Science Foundation grant N 19-72-20067. 


\section{References}

[1] J. A. Hinton, W. Hofmann, Ann. Rev. of Astronomy and Astrophysics 47 (2009) 523

[2] F. A. Aharonian et al., Rep. on Prog. in Physics 71 (2008) 096901

[3] Acharya B., Actis M., Aghajani T. et al, Introducing the CTA concept, Astroparticle Physics 43(2013) 3-18 doi:10.1016/j.astropartphys.2013.01.007.

[4] N. Budnev et al., The TAIGA experiment: From cosmic-ray to gamma-ray astronomy in the Tunka valley, NIM A A845 (2017) 330-333.

[5] M. Tluczykont, D. Hampf, D. Horns and TAIGA col.., Advances in Space Research 48 (2011) 1935

[6] L. Kuzmichev, V. Prosin, C. Spiering, and R. Wischnewski, Astropart. Phys. 56, (2014) 42.

[7] L. Kuzmichev, I.Astapov, P.Bezyazeekov et al, Tunka Advanced Instrument for cosmic rays and Gamma Astronomy (TAIGA): Status, results and perspectives, EPJ Web of Conferences 145 (2017) 01001,doi:10.1051/epjconf/201714501001

[8] L. G. Sveshnikova, I. I. Astapov, P. A. Bezyazeekov, Detecting Gamma Rays with Energies Greater than 3-4 eV from the Crab Nebula and Blazar Markarian 421 by Imaging Atmospheric Cherenkov Telescopes ..., Bull. Russ. Acad. Sc.: Phys. 85 N4 (2021) 398-401

[9] Zhurov D., Gress O., Sidorov D. et al. First results of the tracking system calibration of the TAIGA-IACT telescope, Journal of Physics: Conference Series 1181 (2019) 012045, doi: 10.1088/1742-6596/1181/1/012045.

[10] A.M.Hillas: Space Science Rev. 75 (1996) 17.

[11] J. Albert a, E. Aliu b, H. Anderhub for MAGIC col., Implementation of the Random Forest Method for the Imaging Atmospheric Cherenkov Telescope MAGIC, NIM A 588 (2008) 424 432 doi: 10.1016/j.nima.2007.11.068 .

[12] Grinyuk A., Postnikov E., Sveshnikova L., Monte Carlo Simulation of the TAIGA Hybrid Gamma-Ray Experiment. Physics of Atomic Nuclei 83 N2 (2020) 262-267, doi: 10.1134/S106377882002012X

[13] Postnikov E. B., Kalmykov N. N., Korosteleva E. E. et al, Monte Carlo Simulation of the TAIGA Experiment. Bull. Russ. Acad. Sci.: Physics 83 N8, (2019) 955-958, doi: $10.3103 / \mathrm{S} 1062873819080331$

[14] L. Breimann, J. H. Friedmann, R. A. Olshen, C. J. Stone: Classification and Regression Trees, Wadsworth, 1983. 